\title{
Survival in Patients With Sentinel Node-Positive Melanoma With Extranodal Extension
}

\author{
Andrea Maurichi, MD¹; Francesco Barretta, PhD²; Roberto Patuzzo, $\mathrm{MD}^{1}$; Rosalba Miceli, $\mathrm{PhD}^{2}$; Gianfranco Gallino, $\mathrm{MD}^{1}$;

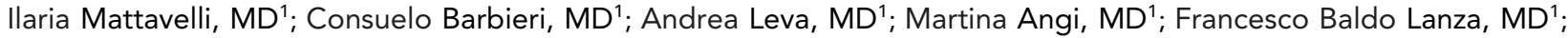

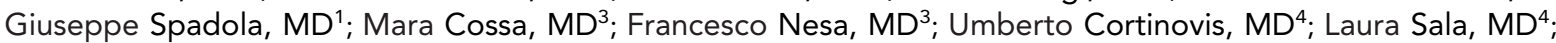

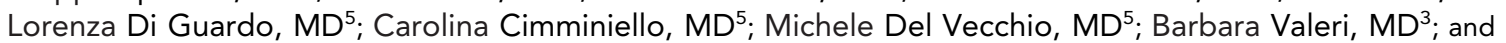
Mario Santinami, $\mathrm{MD}^{1}$

\begin{abstract}
Background: Prognostic parameters in sentinel node (SN)-positive melanoma are important indicators to identify patients at high risk of recurrence who should be candidates for adjuvant therapy. We aimed to evaluate the presence of melanoma cells beyond the SN capsule-extranodal extension (ENE)—as a prognostic factor in patients with positive SNs. Methods: Data from 1,047 patients with melanoma and positive SNs treated from 2001 to 2020 at the Istituto Nazionale dei Tumori in Milano, Italy, were retrospectively investigated. Kaplan-Meier survival and crude cumulative incidence of recurrence curves were estimated. A multivariable logistic model was used to investigate the association between ENE and selected predictive factors. Cox models estimated the effect of the selected predictors on survival endpoints. Results: Median follow-up was 69 months. The 5 -year overall survival rate was $62.5 \%$ and $71.7 \%$ for patients with positive SNs with and without ENE, respectively. The 5 -year disease-free survival rate was $54.0 \%$ and $64.0 \%$ for patients with positive $\mathrm{SNs}$ with and without ENE, respectively. The multivariable logistic model showed that age, size of the main metastatic focus in the SN, and numbers of metastatic non-SNs were associated with ENE (all $P<.0001$ ). The multivariable Cox regression models showed the estimated prognostic effects of ENE associated with age, ulceration, size of the main metastatic focus in the $\mathrm{SN}$, and number of metastatic non-SNs (all $P<.0001$ ) on disease-free survival and overall survival. Conclusions: ENE was a significant prognostic factor in patients with positive-SN melanoma. This parameter may be useful in clinical practice as a selection criterion for adjuvant treatment in patients with stage IIIA disease with a tumor burden $<1 \mathrm{~mm}$ in the SN. We recommend its inclusion as an independent prognostic determinant in future updates of melanoma guidelines.
\end{abstract}

J Natl Compr Canc Netw 2021;19(10):1165-1173 doi: 10.6004/jnccn.2020.7693

\footnotetext{
${ }^{1}$ Melanoma and Sarcoma Surgical Unit, ${ }^{2}$ Clinical Epidemiology and Trial Organization Unit, ${ }^{3}$ Department of Pathology, ${ }^{4}$ Plastic and Reconstructive Surgical Unit, and ${ }^{5}$ Melanoma Medical Oncology Unit, Fondazione IRCCS Istituto Nazionale dei Tumori di Milano, Milan, Italy.
}

\section{Background}

Cutaneous melanoma may spread through the lymphatic circulation to the regional nodal basin, which means that the status of metastatic involvement of the sentinel node ( $\mathrm{SN})$ is one of the most important prognostic markers in melanoma. Nodal metastases are detected in $15 \%$ to $20 \%$ of patients with melanoma who undergo $\mathrm{SN}$ biopsy (SNB). ${ }^{1}$

Until recently, most patients who were SN-positive were offered completion lymph node dissection (CLND) because evidence showed that it could improve prognosis. ${ }^{2}$ However, the Multicenter Selective Lymphadenectomy Trial II and the Dermatologic Cooperative Oncology Group trial showed that immediate CLND did not improve patient survival. ${ }^{3,4}$ As a result, the standard of care changed for patients who are SN-positive, and CLND is no longer performed. Furthermore, systemic treatments for melanoma have also changed, in that novel targeted and immune therapies were shown to have efficacy, improving survival in the adjuvant setting for stage III melanoma, including patients with SN positivity. ${ }^{5-8}$

However, even in a context where novel adjuvant treatments for patients with melanoma are available, SN status remains crucial for staging. ${ }^{9}$ It is important to detect the presence and evaluate the extent of occult metastatic involvement within and beyond the regional nodal basin in order to identify patients at higher risk of progression who may be more carefully evaluated and selected for adjuvant treatments. Some studies have attempted to identify predictors associated with a more aggressive clinical behavior in patients with positive SNs, particularly with regard to metastatic involvement of further non-SNs. Some of these analyses of factors for higher invasiveness focused on characteristics of the primary melanoma and on SN metastasis, ${ }^{10-15}$ including Breslow thickness, presence of

See JNCCN.org for supplemental online content. 
ulceration, a high mitotic rate, location of the metastatic deposit within the SN, size of the largest deposit in the SN, number of foci in the $\mathrm{SN}$, and the positive-to-total SN ratio. ${ }^{10-15}$

More accurate investigation of the regional nodal basin may be useful in patients who are SN-positive with a higher likelihood of developing disease progression. In this regard, risk stratifying patients based on tumor invasion into the perinodal tissues beyond the lymph node capsule - extranodal extension (ENE) — after SNB with a positive result would allow a more appropriate use of adjuvant therapy for those most likely to benefit, while also minimizing the morbidity for patients at lower risk.

Therefore, the aim of the present study was to investigate in patients with SN-positive melanoma, through complete clinicopathologic evaluation of the primary tumor and SN metastasis, whether tumor spread beyond the SN capsule may be associated with an increased risk of disease progression and lower survival probability.

\section{Methods}

\section{Clinicopathologic Characteristics}

A total of 1,047 patients eligible for the study analysis were consecutively treated from 2000 through 2020 at the Istituto Nazionale dei Tumori, in Milano, Italy. The following data were retrieved from a prospectively maintained database: age, sex, primary tumor site, Breslow thickness, ulceration (present vs absent), mitotic rate (mitoses $/ \mathrm{mm}^{2}$ ), Clark level, tumor-infiltrating lymphocytes (absent: no lymphocytes present or lymphocytes present but not infiltrating the tumor; nonbrisk: lymphocytes infiltrating the tumor only focally or not along the entire base of the vertical growth phase; or brisk: lymphocytes diffusely infiltrating the entire base of the vertical growth phase or the entire invasive component of the tumor), lymphovascular invasion (presence vs absence of melanoma cells in lymphatic or blood vessels), tumor regression (present: the replacement of tumor cells with a variable combination of fibrosis, degenerative melanoma cells, melanophages, lymphocytic proliferation, and telangiectasia as a histologic consequence of the interaction between malignant tumor cells and the host immune system; vs absent: when these phenomena were not observed), number of positive SNs, number of metastatic foci in the SN, microanatomic locations of metastatic foci in the SN (subcapsular, parenchymal, combined, multifocal, or extensive), diameter of the main metastatic focus in the $\mathrm{SN}$, and number of further positive non-SNs after CLND.

Pathologic criteria for classifying the metastatic deposit within each SN were as follows: subcapsular (ie, confined to the subcapsular sinus), parenchymal (ie, entirely within the paracortical area of the parenchyma), combined (ie, subcapsular and parenchymal metastasis), multifocal (ie, multiple metastatic deposits), and extensive (ie, any metastasis $>5 \mathrm{~mm}$ or any node with extracapsular spread). All slides were reviewed independently by a pool of dermatopathologists (B. Valeri, M. Cossa, and F. Nesa) according to a common protocol, ${ }^{16}$ with disagreements being resolved by discussion. Diagnosis and staging of all cases were revised according to the criteria of the 8th edition of the AJCC Cancer Staging Manual. ${ }^{9}$ All patients underwent an initial diagnostic biopsy followed by a wider excision- 1 versus $2 \mathrm{~cm}$, depending on the Breslow thickness ${ }^{17}$-and SNB. All surgical treatments were performed after the benefits and potential harms were discussed with the patient and informed consent was obtained. Pathologic assessment of SNs was performed according to the EORTC protocol. ${ }^{18}$ Patients with positive SNs were offered CLND as additional therapy.

\section{Statistical Methods}

The primary aim of this study was to compare diseasefree survival (DFS) and overall survival (OS) between patients with and without ENE. Survival times were calculated from the date of primary surgery. DFS was defined as the time to recurrence or death, whichever occurred first. OS was defined as the time to death from any cause. Time was censored for living patients who were free from events at last follow-up. OS and DFS curves were estimated using the Kaplan-Meier method and compared using the log-rank test. Median follow-up was estimated with the reverse Kaplan-Meier method using OS data. ${ }^{19}$

The crude cumulative incidence (CCI) of local relapse, regional relapse, and distant metastasis was estimated in a competing risk setting using cumulative incidence estimates; the curves were compared using the weighted Gray test. In the estimation of the CCIs, events other than estimated relapse and death without relapse were evaluated as competing events.

Differences in demographic and clinicopathologic characteristics between study groups were assessed by means of the standardized mean difference (SMD).$^{20}$ SMD is a continuous measure of the magnitude of the mean differences, its value ranging from 0 to infinite and increasing with the increase in mean differences. It is considered indicative of a possible between-group imbalance when approaching a value of approximately 0.3 ; however, the significance of SMD should be evaluated from a clinical point of view.

Univariable and multivariable logistic models including selected covariates were performed to assess their association with ENE, with an odds ratio $(\mathrm{OR})>1$ indicating an increased OR of ENE. Univariable Cox models were performed before 2 backward selection procedures based on Akaike Information Criteria were applied to the same set of covariates to select the prognostic factors for OS and DFS. 


\section{Table 1. Clinicopathologic Characteristics of SN-Positive Patients}

\begin{tabular}{|c|c|c|c|c|}
\hline & $\begin{array}{l}\text { Overall } \\
\text { n (\%) }\end{array}$ & $\begin{array}{l}\text { ENE } \\
\text { n (\%) }\end{array}$ & $\begin{array}{c}\text { No ENE } \\
\text { n (\%) }\end{array}$ & SMD \\
\hline Patients, $\mathrm{n}$ & 1,047 & 148 & 899 & \\
\hline Female & $601(57.4)$ & $88(59.5)$ & $513(57.1)$ & \\
\hline Male & $446(42.6)$ & $60(40.5)$ & $386(42.9)$ & \\
\hline Site of primary melanoma & & & & 0.159 \\
\hline Lower limbs & $336(32.1)$ & $42(28.4)$ & $294(32.7)$ & \\
\hline Upper limbs & $112(10.7)$ & $19(12.8)$ & $93(10.3)$ & \\
\hline Genitals & $1(0.1)$ & $1(0.7)$ & $0(0.0)$ & \\
\hline Median (1st and 3rd quartile) & $2.70(1.60-4.32)$ & $4.00(2.50-7.00)$ & $2.50(1.52-4.00)$ & \\
\hline Clark level & & & & 0.459 \\
\hline$\|$ & $6(0.6)$ & $0(0.0)$ & $6(0.7)$ & \\
\hline III & $110(10.5)$ & $6(4.1)$ & $104(11.6)$ & \\
\hline IV & $846(80.8)$ & $115(77.7)$ & $731(81.3)$ & \\
\hline $\mathrm{v}$ & $85(8.1)$ & $27(18.2)$ & $58(6.5)$ & \\
\hline Mitoses $/ \mathrm{mm}^{2}$ & & & & 0.182 \\
\hline Median (1st and 3rd quartile) & $5.00(2.00-7.00)$ & $6.00(3.00-8.00)$ & $4.00(2.00-7.00)$ & \\
\hline Tumor-infiltrating lymphocytes & & & & 0.181 \\
\hline Not evaluable & $14(1.3)$ & $1(0.7)$ & $13(1.4)$ & \\
\hline Lymphovascular invasion & & & & 0.234 \\
\hline Absent & $861(82.2)$ & $112(75.7)$ & $749(83.3)$ & \\
\hline Present & $142(13.6)$ & $31(20.9)$ & $111(12.3)$ & \\
\hline Not evaluable & $44(4.2)$ & $5(3.4)$ & $39(4.3)$ & \\
\hline Ulceration & & & & 0.412 \\
\hline Absent & $570(54.4)$ & $56(37.8)$ & $514(57.2)$ & \\
\hline Present & $473(45.2)$ & $92(62.2)$ & $381(42.4)$ & \\
\hline Not evaluable & $4(0.4)$ & $0(0.0)$ & $4(0.4)$ & \\
\hline Number of positive SNs (continuous) & & & & 0.339 \\
\hline Median (1st and 3rd quartile) & $1.00(1.00-1.00)$ & $1.00(1.00-2.00)$ & $1.00(1.00-1.00)$ & \\
\hline Number of positive SNs & & & & 0.381 \\
\hline 1 & $862(82.3)$ & $102(68.9)$ & $760(84.5)$ & \\
\hline 2 & $152(14.5)$ & $36(24.3)$ & $116(12.9)$ & \\
\hline 3 & $24(2.3)$ & $7(4.7)$ & $17(1.9)$ & \\
\hline$>3$ & $9(0.9)$ & $3(2.0)$ & $6(0.7)$ & \\
\hline
\end{tabular}




\begin{tabular}{|c|c|c|c|c|}
\hline & $\begin{array}{c}\text { Overall } \\
\text { n (\%) }\end{array}$ & $\begin{array}{l}\text { ENE } \\
\text { n (\%) }\end{array}$ & $\begin{array}{c}\text { No ENE } \\
\text { n (\%) }\end{array}$ & SMD \\
\hline \multicolumn{4}{|c|}{ Number of positive non-SNs after CLND (continuous) } & 0.482 \\
\hline Median (1st and 3rd quartile) & $0.00(0.00-0.00)$ & $0.00(0.00-2.00)$ & $0.00(0.00-0.00)$ & \\
\hline \multicolumn{4}{|c|}{ Number of positive non SNs after CLND } & 0.748 \\
\hline 0 & $765(80.7)$ & $76(54.7)$ & $689(85.2)$ & \\
\hline 1 & $91(9.6)$ & $23(16.5)$ & $68(8.4)$ & \\
\hline 2 & $45(4.7)$ & $14(10.1)$ & $31(3.8)$ & \\
\hline $3-5$ & $20(2.1)$ & $9(6.5)$ & $11(1.4)$ & \\
\hline $6-8$ & $14(1.5)$ & $7(5.0)$ & $7(0.9)$ & \\
\hline$>8$ & $13(1.4)$ & $10(7.2)$ & $3(0.4)$ & \\
\hline \multicolumn{4}{|l|}{ Number of metastatic foci in SN } & 0.165 \\
\hline Median (1st and 3rd quartile) & $2.00(1.00-3.00)$ & $2.00(1.00-3.00)$ & $1.00(1.00-3.00)$ & \\
\hline \multicolumn{4}{|c|}{ Location of main metastatic focus in $\mathrm{SN}$} & 0.640 \\
\hline Combined & $48(4.6)$ & $6(4.1)$ & $42(4.7)$ & \\
\hline Extensive & $24(2.3)$ & $18(12.2)$ & $6(0.7)$ & \\
\hline Multifocal & $13(1.2)$ & $3(2.0)$ & $10(1.1)$ & \\
\hline Parenchymal & $655(62.6)$ & $101(68.2)$ & $554(61.6)$ & \\
\hline Subcapsular sinus & $303(28.9)$ & $20(13.5)$ & $283(31.5)$ & \\
\hline Not evaluable & $4(0.4)$ & $0(0.0)$ & $4(0.4)$ & \\
\hline \multicolumn{4}{|c|}{ Size of main metastatic focus in $\mathrm{SN}, \mathrm{mm}$} & 1.099 \\
\hline Median (1st and 3rd quartile) & $1.00(0.30-3.00)$ & $5.50(2.58-13.00)$ & $0.70(0.20-2.23)$ & \\
\hline
\end{tabular}

Abbreviations: CLND, completion lymph node dissection; ENE, extranodal extension; SMD, standardized mean difference; SN, sentinel node.

In addition, the interaction terms of all factors with ENE were tested. Two Cox models including the selected covariates were applied to estimate their effects on prognosis. All continuous variables were modeled using 3-knot restricted cubic splines ${ }^{21}$ to allow nonlinear relationships with the outcomes through the estimation of piecewise polynomial regressions within predefined values of the explanatory variable (knots), imposing continuity between them and linearity on both tails (before the first knot and after the last one) to control their natural instability. ${ }^{21}$ Nonlinearity may result in curves of any shape, and the interpretation cannot be straightforward as in a linear model because the effect of a covariate could be different in different intervals of the same length. The analyses were performed using SAS version 9.2 (SAS Institute Inc) and the R software version 3.5.2 (R Foundation for Statistical Computing).

\section{Results}

Characteristics of Patients, Primary Tumors, and SNs Of the 1,047 patients who were SN-positive, 148 (14.1\%) showed ENE and 899 (85.9\%) did not (Table 1). Patients with and without ENE did not differ by sex, primary tumor site, mitoses $/ \mathrm{mm}^{2}$, tumor-infiltrating lymphocytes, tumor regression, and number of metastatic foci in the SN. Median age was 63.5 and 54.0 years in the groups with and without ENE, respectively (SMD, 0.417), and median Breslow thickness was 4.0 and $2.5 \mathrm{~mm}$ in patients with and without ENE, respectively (SMD, 0.493). Of patients with SN-positive melanomas, ulceration was present in $62.2 \%$ with ENE compared with $42.4 \%$ without ENE (SMD, 0.412). The group with ENE had higher numbers of positive SNs (SMD, 0.381) and non-SNs after CLND (SMD, 0.748 ) and a larger-sized main metastatic focus in the $\mathrm{SN}$ compared with the group without ENE (SMD, 1.099).

\section{Association Analysis}

In the univariable logistic models, all selected covariates were statistically associated with ENE except the number of metastatic foci in the SN. In particular, older age, ulceration of the primary tumor, greater number of positive SNs, location of the main metastatic focus in the SN, size of the main metastatic focus in the SN, and number of further positive non-SNs after CLND were associated with a higher probability of ENE. In the multivariable model only, age (OR, 1.80; 95\% CI, $1.27-2.56 ; P=.001$ ), size of the main metastatic focus in the SN (OR, 9.99; 95\% CI, $5.55-17.96$; $P<.001$ ), and number of further positive non-SNs after CLND (OR, $1.66[95 \%$ CI, $0.88-3.14] ; 1.82[95 \% \mathrm{CI}$, $0.81-4.08] ; 2.95 \quad[95 \%$ CI, $0.99-8.78] ; 8.17 \quad[95 \%$ CI, 2.06-32.45]; and 7.42 [95\% CI, 1.79-30.78] for 1, 2, 3-5, 6-8, 
and $>8$ positive non-SNs after CLND, respectively; $P=.001$ ) kept their statistical significance, indicating a relevant role even after adjustment. The number of metastatic foci in the SN had a higher OR (1.74; 95\% CI, 1.03-2.94) and maintained its $P$ value (.074) in the multivariable model (Table 2).

\section{Prognostic Models}

In the univariable Cox models, all assessed covariates except metastatic foci in the SN were statistically significant (supplemental eTable 1, available with this article at JNCCN.org). The final models for both OS and DFS included ENE, patient age, ulceration of the primary tumor, size of the main metastatic focus in the $\mathrm{SN}$, and number of further positive non-SNs after CLND as significant prognostic covariates (all $P<.001$ ). The effect of ENE was different (indicative of interaction) at different ages in both models. In particular, in younger patients, ENE was estimated to be an unfavorable prognostic factor (hazard ratio [HR], 2.17; 95\% CI, 0.78-6.04 vs HR, 0.78; 95\% CI, 0.51-1.17; and $\mathrm{HR}, 2.00$; 95\% CI, 0.84-4.74 vs HR, 0.39; 95\% CI, $0.24-0.64$ at age 25 vs 65 years in the OS and DFS models, respectively) (Table 3 ).

In the DFS model, among patients with ENE, the number of positive lymph nodes at dissection had a smaller and more constant, unfavorable prognostic effect (nonlinear HR range, 1.22-2.73), whereas in the absence of ENE there was a greater number of further positive non-SNs after CLND (HR, 0.99, 3.06, 3.94, 5.43, and 1.92 for $1,2,3-5,6-8$, and $>8$ positive lymph nodes, respectively, vs 0 ) (Table 3 ).

\section{Survival Comparison}

Median follow-up was 81 months (interquartile range [IQR], 39-153 months) and 69 months (IQR, 31-110 months) in patients with and without ENE, respectively. Rates of 5- and 10-year OS were $62.5 \% \quad(95 \%$ CI,

\section{Table 2. Results of Univariable and Multivariable Logistic Models for Estimation of OR of ENE}

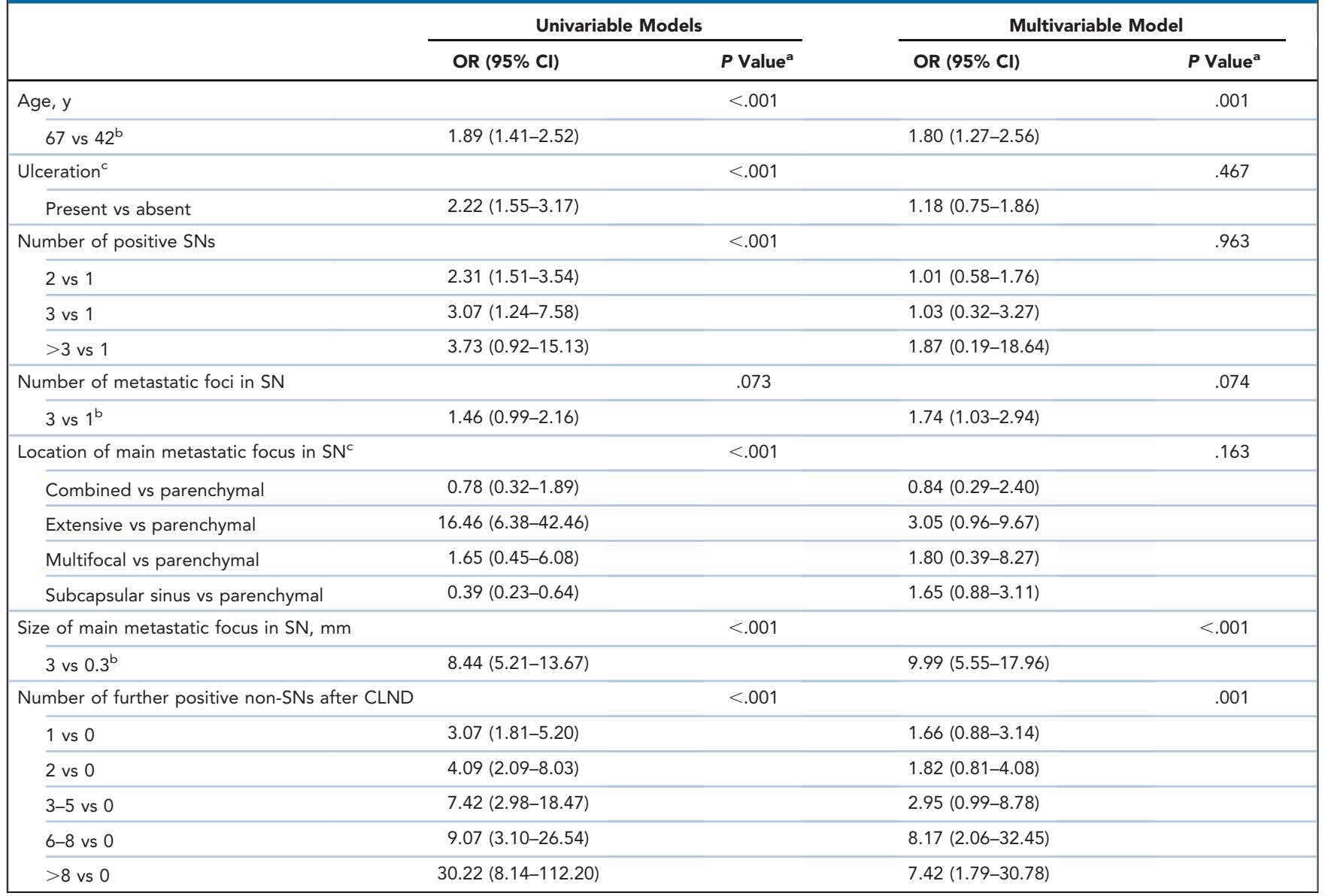

Abbreviations: CLND, completion lymph node dissection; ENE, extranodal extension; OR, odds ratio; SN, sentinel node.

${ }^{a}$ Calculated using a 2-sided Wald test.

${ }^{\mathrm{b}}$ Modeled as restricted cubic spline; values representing $3 \mathrm{rd}$ and 1 st quartiles.

'Patients in whom ulceration and location of the main metastatic focus in the SN were not evaluable were excluded from the analyses. 


\begin{tabular}{|c|c|c|c|c|}
\hline & \multicolumn{2}{|c|}{ os } & \multicolumn{2}{|c|}{ DFS } \\
\hline & $\mathrm{HR}(95 \% \mathrm{Cl})$ & $P$ Value $^{\mathrm{a}}$ & $\mathrm{HR}(95 \% \mathrm{Cl})$ & $P$ Value ${ }^{a}$ \\
\hline ENE & & $<.001$ & & $<.001$ \\
\hline At age $25 y$ & $2.17(0.78-6.04)$ & & $2.00(0.84-4.74)$ & \\
\hline At age $45 y$ & $0.93(0.58-1.49)$ & & $0.74(0.46-1.20)$ & \\
\hline At age $65 \mathrm{y}$ & $0.78(0.51-1.17)$ & & $0.39(0.24-0.64)$ & \\
\hline At number of positive nodes at dissection $=0$, age $25 \mathrm{y}$ & - & & $2.00(0.84-4.74)$ & \\
\hline At number of positive nodes at dissection $=2$, age $25 \mathrm{y}$ & - & & $1.53(0.49-4.76)$ & \\
\hline At number of positive nodes at dissection $=6-8$, age $25 \mathrm{y}$ & - & & $0.45(0.09-2.18)$ & \\
\hline Age, y & & $<.001$ & & $<.001$ \\
\hline 67 vs $42^{\mathrm{b}}$ without ENE & $1.87(1.50-2.33)$ & & $1.74(1.45-2.08)$ & \\
\hline 67 vs $42^{b}$ with ENE & $0.78(0.51-1.17)$ & & $0.78(0.55-1.12)$ & \\
\hline Ulceration & & $<.001$ & & $<.001$ \\
\hline Present vs absent & $2.21(1.72-2.85)$ & & $2.22(1.80-2.73)$ & \\
\hline Size of main metastatic focus in $\mathrm{SN}, \mathrm{mm}$ & & $<.001$ & & $<.001$ \\
\hline 3 vs $0.3^{b}$ & $2.06(1.58-2.69)$ & & $1.99(1.59-2.49)$ & \\
\hline Number of further positive non-SNs after CLND, age $55 \mathrm{y}$ & & $<.001$ & & $<.001$ \\
\hline 1 vs 0 without ENE & - & & $0.99(0.69-1.42)$ & \\
\hline 2 vs 0 without ENE & - & & $3.06(1.88-4.97)$ & \\
\hline $3-5$ vs 0 without ENE & - & & $3.94(2.06-7.55)$ & \\
\hline $6-8$ vs 0 without ENE & - & & $5.43(2.21-13.31)$ & \\
\hline$>8$ vs 0 without ENE & - & & $1.92(0.60-6.17)$ & \\
\hline 1 vs 0 with ENE & $1.01(0.68-1.48)$ & & $1.30(0.68-2.49)$ & \\
\hline 2 vs 0 with ENE & $2.87(1.83-4.50)$ & & $2.34(1.16-4.71)$ & \\
\hline $3-5$ vs 0 with ENE & $3.35(1.89-5.93)$ & & $2.03(0.84-4.94)$ & \\
\hline $6-8$ vs 0 with ENE & $2.05(0.87-4.84)$ & & $1.22(0.42-3.52)$ & \\
\hline$>8$ vs 0 with ENE & $2.40(1.17-4.90)$ & & $2.73(1.26-5.90)$ & \\
\hline
\end{tabular}

Abbreviations: CLND, completion lymph node dissection; DFS, disease-free survival; ENE, extranodal extension; HR, hazard ratio; OS, overall survival; SN, sentinel node.

${ }^{a}$ Calculated using a 2-sided Wald test.

brd vs 1 st quartile.

$54.3 \%-72.0 \%)$ and $47.5 \%$ (95\% CI, $38.2 \%-59.1 \%)$ in patients with ENE and $71.7 \%(95 \% \mathrm{CI}, 68.3 \%-75.3 \%)$ and $60.8 \%$ (95\% CI, 56.6\%-65.3\%) in patients without ENE, respectively (Figure 1). Rates of 5- and 10-year DFS were $54.0 \%(95 \%$ CI, $45.7 \%-63.8 \%)$ and $33.7 \%$ (95\% CI, $25.4 \%-44.8 \%)$ in patients with ENE and $64.0 \%(95 \% \mathrm{CI}$, $60.5 \%-67.7 \%$ ) and $45.2 \%$ (95\% CI, $40.9 \%-49.9 \%$ ) in patients without ENE, respectively (Figure 2A). The log-rank tests showed statistically significantly better survival (both OS and DFS) for patients without ENE $(P<.001$ and $P=.007$, respectively). No differences were observed in the CCIs of local and regional relapse (Figure 2B, C), whereas a significant difference $(P<.001)$ was observed in the 5- and 10-year CCI of distant metastasis: $31.8 \%$ (95\% CI, $24.4 \%-41.4 \%$ ) and $47.1 \%(95 \%$ CI, $38.2 \%-58.1 \%)$ in patients with ENE and $22.2 \%(95 \% \mathrm{CI}, 19.3 \%-25.6 \%)$ and $30.0 \%(95 \% \mathrm{CI}$, $26.4 \%-34.2 \%$ ) in those without ENE (Figure 2D).

\section{Discussion}

The main result of our analyses was that patients with SN-positive melanoma with metastatic cells beyond the SN capsule had significantly worse 10-year OS than patients who were SN-positive without ENE (47.5\% [95\% CI, $38.2 \%-59.1 \%$ ] vs $60.8 \%$ [95\% CI, $56.6 \%-65.3 \%]$ ). In addition, 10-year DFS showed significant differences between patients who were SN-positive with and without ENE $(33.7 \%$ [95\% CI, $25.4 \%-44.8 \%$ ] vs $45.2 \%$ [95\% CI, $40.9 \%-49.9 \%]$ ). This large study is the first ever performed focusing on the prognostic role of ENE specifically in patients with SN-positive melanoma. Previous analyses were conducted to selectively investigate extranodal spread as an adverse prognostic factor in stage III melanoma $^{22,23}$; however, those studies analyzed either a heterogeneous group comprising all patients with stage III disease, including those with unknown primary 


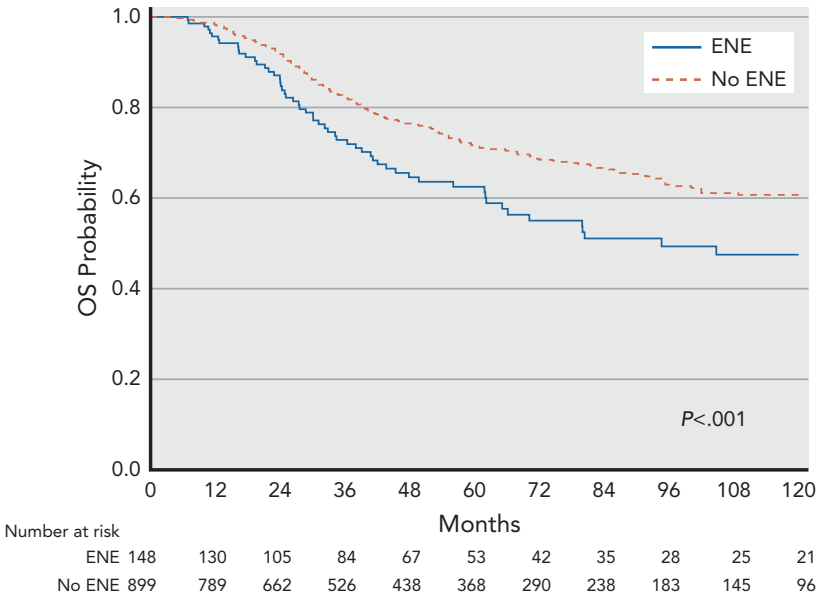

Figure 1. Kaplan-Meier OS curves according to the presence of ENE. Abbreviations: $E N E$, extranodal extension; OS, overall survival.

melanoma, ${ }^{22}$ or patients with clinically detected lymph node metastases. ${ }^{23}$ Other studies have shown either discordant results about how the role of ENE in affecting survival may be quantified, or insufficient data to support
ENE as a candidate factor for inclusion in adjuvant clinical trials. ${ }^{24-26}$ Some studies investigated factors predictive of a higher risk of further metastatic non-SNs in patients with SN-positive melanoma, and indicated SN tumor burden as the most important prognostic factor in these patients, whose outcome also correlated with other characteristics of the metastases in the $\mathrm{SN}$, such as size and site. ${ }^{10-12}$ In those reports, all investigated factors reflected different biologic behaviors and helped identify patients who may or may not need additional locoregional and/or systemic therapy ${ }^{10-12}$ However, none of the studies considered melanoma metastasis beyond the SN capsule as a further prognostic factor to be investigated. ${ }^{10-12}$

In our series, the percentage of patients with ENE was $>14 \%$, a nonnegligible portion of the SN-positive population, making this variable worthy of a specific analysis regarding its possible prognostic role. Furthermore, although in the last AJCC staging manual the definition of ENE was not formally included, the AJCC also recommended that this factor be recorded, because it could prove useful for future analyses. ${ }^{9}$ Our data also showed that in younger patients, ENE was a worse prognostic

A

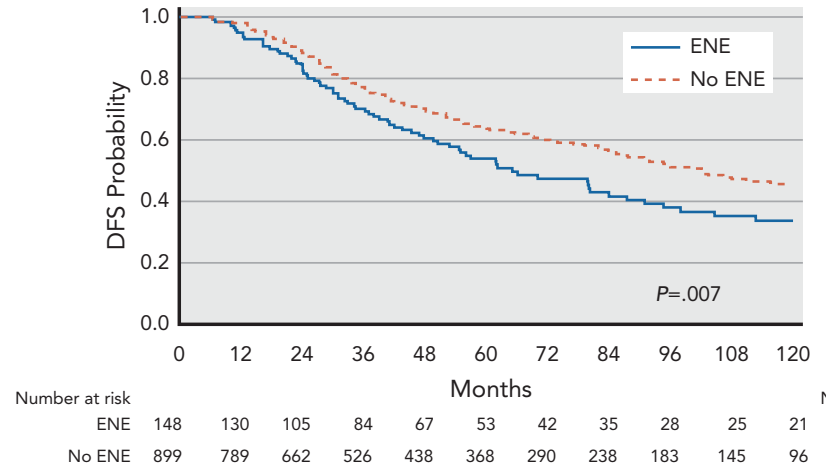

B

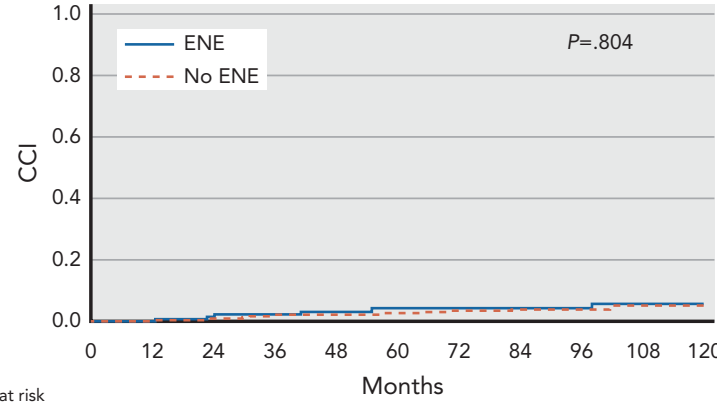

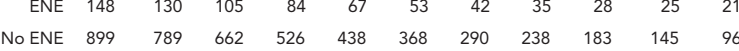

$\begin{array}{llllllllllll}\text { ENE } & 148 & 130 & 105 & 84 & 67 & 53 & 42 & 35 & 28 & 25 & 21\end{array}$

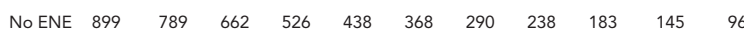

C
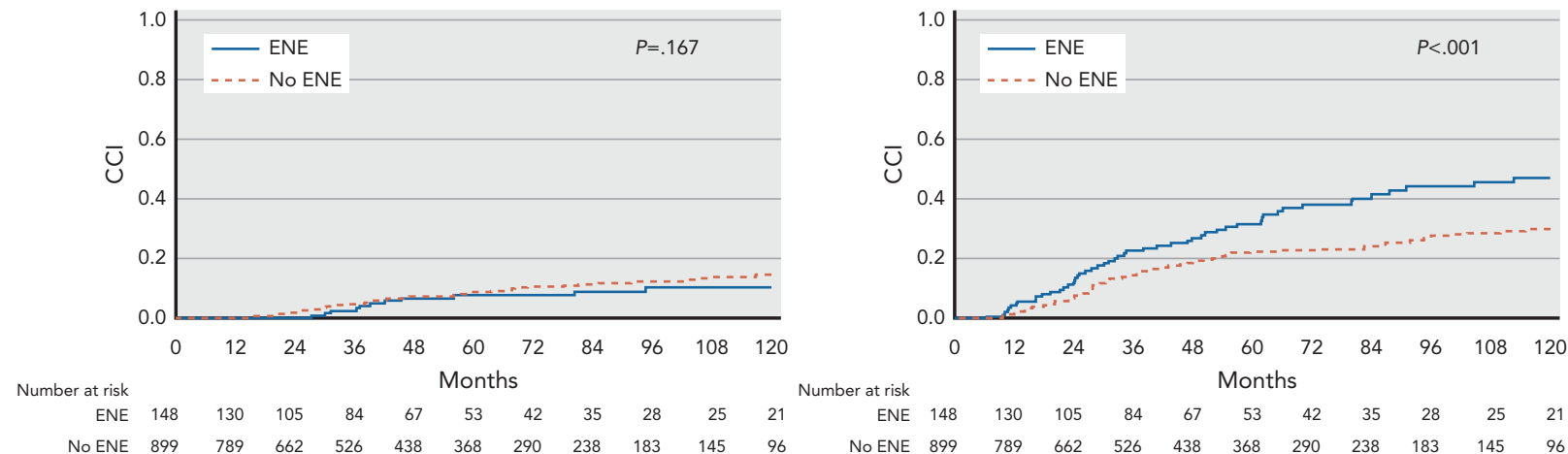

Figure 2. Kaplan-Meier DFS curves (A), and CCl curves of local relapse (B), regional relapse (C), and distant metastasis (D) according to the presence of ENE.

Abbreviations: $\mathrm{CCl}$, crude cumulative incidence; DFS, disease-free survival; $\mathrm{ENE}$, extranodal extension. 
factor for both OS and DFS; this finding may allow a more accurate risk stratification and facilitate decisions for activating adjuvant therapies and individualizing management and follow-up.

Another important finding from our analyses was that the presence of ENE reduced the prognostic value of the concomitant presence of further positive non-SNs, irrespective of the number of metastatic nodes; conversely, the absence of ENE reinstated the prognostic role of an increasing number of further positive non-SNs. This finding highlights that ENE represents the expression of disease developing beyond the locoregional district and therefore distant disease (stage IV, Mla). This hypothesis was supported by our data showing that ENE was associated with a higher incidence of distant metastasis. ENE should therefore be considered for inclusion in the AJCC staging system as an important parameter affecting the transition from stage III to stage IV disease. Patient age, size of the main metastatic focus in the $\mathrm{SN}$, and number of further positive non-SNs have been reported as established predictors of recurrence. ${ }^{27}$ In our study, these 3 parameters maintained statistical significance also as predictors of the presence of ENE.

It seems important to appropriately quantify the extent of $\mathrm{ENE}^{28}$ for supporting further clinical decisions in all patients who may be candidates for surgical debulking in order to benefit from more effective local disease control. In our study, the high incidence of distant recurrences and the worse survival observed among patients who were $\mathrm{SN}$ positive with ENE emphasizes the role of adjuvant therapies to prevent the occurrence of distant metastases. ${ }^{6,29}$ Presence of ENE may be useful in clinical practice as a selection criterion for offering adjuvant treatments to patients with stage IIIA disease with a tumor burden $<1 \mathrm{~mm}$ in the SN. This clinical approach is in accord with the current ASCO guidelines, ${ }^{30}$ which emphasize that these patients should receive individualized treatment after discussion of the risk/benefit quotient with their physician.

The strengths of our study are (1) the inclusion of a large cohort of patients to address the issue of a possible prognostic role for ENE in patients who are SN-positive; (2) the collection of data in a specific database from a comprehensive cancer center with information on demographics, diagnosis, surgical procedures, and histopathological characteristics; and (3) long-term follow-up allowing the investigation of mature data on DFS, OS, and the incidence of distant metastasis. The main limitation concerns the retrospective nature of our analysis, which cannot exclude patient enrollment bias.

\section{Conclusions}

Our data showed that ENE was a highly significant independent adverse prognostic parameter for recurrence and survival in a large series of patients with stage III SNpositive melanoma. We suggest that ENE should be included in the next AJCC melanoma staging manual and also in future updates of melanoma guidelines to improve the stratification of patients in terms of recurrence risk and to support clinical decisions in the context of ever-greater personalized management of care.

\section{Acknowledgments}

The authors thank Marije de Jager for help with the English in this article. Antonio Florita and Elena Morittu are acknowledged for assistance in manuscript submission.

Submitted July 9, 2020; final revision received November 5, 2020; accepted for publication November 30, 2020.

Published online July 26, 2021.

Author contributions: Study concept: Maurichi, Barretta, Miceli, Santinami. Data acquisition: Patuzzo, Gallino, Mattavelli, Barbieri, Leva, Spadola, Cortinovis, Sala. Data analysis and interpretation: Maurichi, Cossa, Nesa Cimminiello, Di Guardo, Valeri, Santinami. Statistical analysis: Barretta, Miceli. Manuscript preparation: Maurichi, Barretta, Miceli, Angi, Lanza, Del Vecchio. Manuscript review and editing: All authors.

Disclosures: The authors have disclosed that they have not received any financial considerations from any person or organization to support the preparation, analysis, results, or discussion of this article.

Correspondence: Andrea Maurichi, MD, Melanoma and Sarcoma Unit, Fondazione IRCCS Istituto Nazionale dei Tumori di Milano, Via Giacomo Venezian 1, 20133 Milan, Italy. Email: andrea.maurichi@istitutotumori.mi.it

\section{References}

1. Morton DL, Thompson JF, Cochran AJ, et al. Sentinel-node biopsy or nodal observation in melanoma. N Engl J Med 2006:355:1307-1317.

2. Coit DG, Andtbacka R, Bichakjian CK, et al. NCCN Clinical Practice Guidelines in Oncology: Melanoma. Version 2.2009. To view the most recent version, visit NCCN.org

3. Faries MB, Thompson JF, Cochran AJ, et al. Completion dissection or observation for sentinel-node metastasis in melanoma. N Engl J Med 2017;376:2211-2222.

4. Leiter U, Stadler R, Mauch C, et al. Final analysis of DeCOG-SLT trial: no survival benefit for complete lymph node dissection in patients with melanoma with positive sentinel node. J Clin Oncol 2019;37:3000-3008.

5. Eggermont AM, Chiarion-Sileni V, Grob JJ, et al. Adjuvant ipilimumab versus placebo after complete resection of high-risk stage III melanoma
(EORTC 18071): a randomised, double-blind, phase 3 trial. Lancet Oncol 2015;16:522-530.

6. Long GV, Hauschild A, Santinami M, et al. Adjuvant dabrafenib plus trametinib in stage III BRAF-mutated melanoma. N Engl J Med 2017;377:1813-1823.

7. Maio M, Lewis K, Demidov L, et al. Adjuvant vemurafenib in resected, $\mathrm{BRAF}^{\mathrm{V} 600}$ mutation-positive melanoma (BRIM8): a randomised, doubleblind, placebo-controlled, multicentre, phase 3 trial. Lancet Oncol 2018;19:510-520.

8. Weber J, Mandala M, Del Vecchio M, et al. Adjuvant nivolumab versus ipilimumab in resected stage III or IV melanoma. N Engl J Med 2017;377:1824-1835.

9. Gershenwald JE, Scolyer RA, Hess KR, et al. Melanoma staging: evidencebased changes in the American Joint Committee on Cancer eighth edition cancer staging manual. CA Cancer J Clin 2017;67:472-492. 
10. Cadili A, Scolyer RA, Brown PT, et al. Total sentinel lymph node tumor size predicts nonsentinel node metastasis and survival in patients with melanoma. Ann Surg Oncol 2010;17:3015-3020.

11. Dewar DJ, Newell B, Green MA, et al. The microanatomic location of metastatic melanoma in sentinel lymph nodes predicts nonsentinel lymph node involvement. J Clin Oncol 2004;22:3345-3349.

12. Gershenwald JE, Andtbacka RH, Prieto VG, et al. Microscopic tumor burden in sentinel lymph nodes predicts synchronous nonsentinel lymph node involvement in patients with melanoma. J Clin Oncol 2008;26:4296-4303.

13. Lee $\mathrm{JH}$, Essner $\mathrm{R}$, Torisu-Itakura $\mathrm{H}$, et al. Factors predictive of tumor-positive nonsentinel lymph nodes after tumor-positive sentinel lymph node dissection for melanoma. J Clin Oncol 2004;22:3677-3684.

14. Murali R, Desilva C, Thompson JF, et al. Non-Sentinel Node Risk Score (N-SNORE): a scoring system for accurately stratifying risk of non-sentine node positivity in patients with cutaneous melanoma with positive sentinel lymph nodes. J Clin Oncol 2010;28:4441-4449.

15. van der Ploeg AP, van Akkooi AC, Rutkowski P, et al. Prognosis in patients with sentinel node-positive melanoma is accurately defined by the combined Rotterdam tumor load and Dewar topography criteria. J Clin Oncol 2011;29:2206-2214.

16. Frishberg DP, Balch C, Balzer BL, et al. Protocol for the examination of specimens from patients with melanoma of the skin. Arch Pathol Lab Med 2009;133:1560-1567.

17. Michielin O, van Akkooi ACJ, Ascierto PA, et al. Cutaneous melanoma: ESMO clinical practice guidelines for diagnosis, treatment and follow-up. Ann Oncol 2019;30:1884-1901.

18. Cook MG, Massi D, Szumera-Cieckiewicz A, et al. An updated European Organisation for Research and Treatment of Cancer (EORTC) protocol for pathological evaluation of sentinel lymph nodes for melanoma. Eur J Cancer 2019;114:1-7.

19. Schemper M, Smith TL. A note on quantifying follow-up in studies of failure time. Control Clin Trials 1996;17:343-346.

20. Flury BK, Reidwyl H. Standard distance in univariate and multivariate analysis. Am Stat 1986;40:249-251.
21. Durrleman S, Simon R. Flexible regression models with cubic splines. Stat Med 1989;8:551-561.

22. Crookes TR, Scolyer RA, Lo $\mathrm{S}$, et al. Extranodal spread is associated with recurrence and poor survival in stage III cutaneous melanoma patients. Ann Surg Oncol 2017;24:1378-1385.

23. Koopal SA, Tiebosch AT, Daryanani D, et al. Extra nodal growth as a prognostic factor in malignant melanoma. Eur J Surg Oncol 2005:31:88-94.

24. Spillane AJ, Pasquali S, Haydu LE, et al. Patterns of recurrence and survival after lymphadenectomy in melanoma patients: clarifying the effects of timing of surgery and lymph node tumor burden. Ann Surg Oncol 2014:21:292-299.

25. Burmeister $\mathrm{BH}$, Henderson MA, Ainslie J, et al. Adjuvant radiotherapy versus observation alone for patients at risk of lymph-node field relapse after therapeutic lymphadenectomy for melanoma: a randomised trial. Lancet Oncol 2012;13:589-597.

26. van der Ploeg AP, van Akkooi AC, Haydu LE, et al. The prognostic significance of sentinel node tumour burden in melanoma patients: an international, multicenter study of 1539 sentinel node-positive melanoma patients. Eur J Cancer 2014;50:111-120.

27. Balch $\mathrm{CM}$, Gershenwald JE, Soong SJ, et al. Multivariate analysis of prognostic factors among 2,313 patients with stage III melanoma: comparison of nodal micrometastases versus macrometastases. J Clin Oncol 2010;28:2452-2459

28. Murali R, Cochran AJ, Cook MG, et al. Interobserver reproducibility of histologic parameters of melanoma deposits in sentinel lymph nodes: implications for management of patients with melanoma. Cancer 2009:115:5026-5037.

29. Eggermont AMM, Blank CU, Mandala M, et al. Adjuvant pembrolizumab versus placebo in resected stage III melanoma. N Engl J Med 2018;378:1789-1801.

30. Seth R, Messersmith $H$, Kaur V, et al. Systemic therapy for melanoma: ASCO guideline. J Clin Oncol 2020;38:3947-3970.
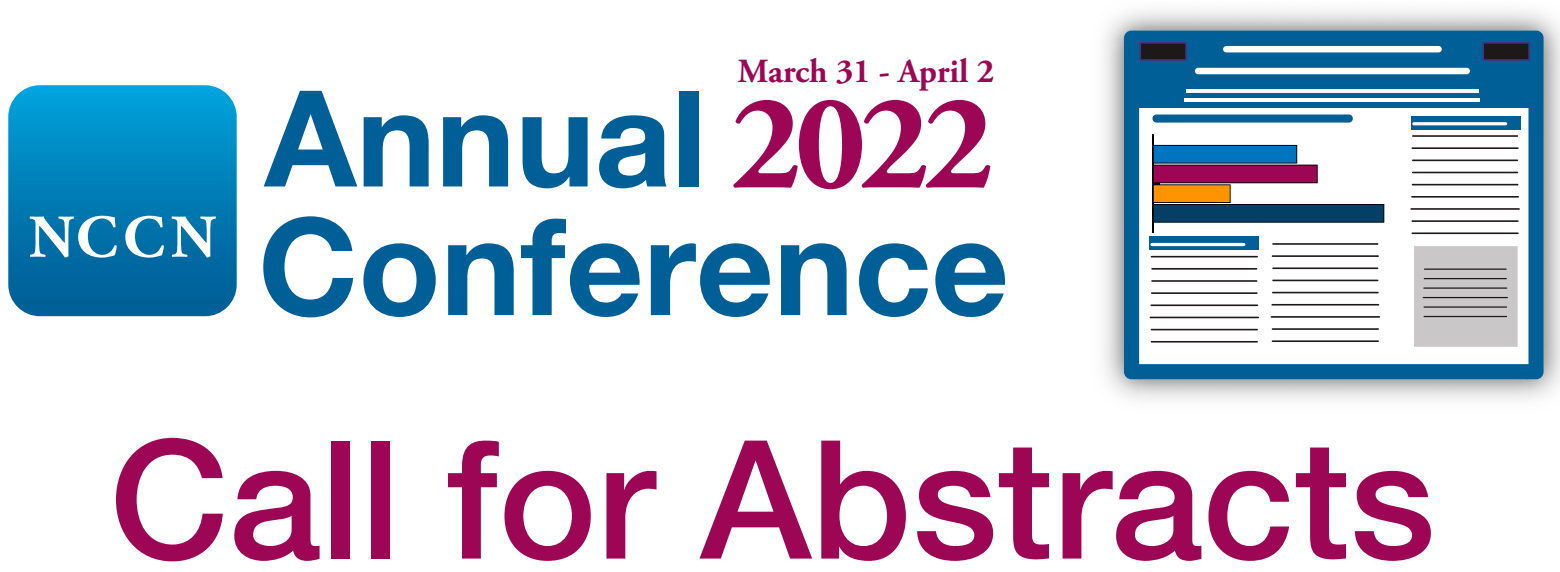

Deadline is November 10!

Submit now: NCCN.org/conference 
Supplemental online content for:

\section{Survival in Patients With Sentinel Node-Positive Melanoma With Extranodal Extension}

Andrea Maurichi, MD; Francesco Barretta, PhD; Roberto Patuzzo, MD; Rosalba Miceli, PhD;

Gianfranco Gallino, MD; Ilaria Mattavelli, MD; Consuelo Barbieri, MD; Andrea Leva, MD;

Martina Angi, MD; Francesco Baldo Lanza, MD; Giuseppe Spadola, MD; Mara Cossa, MD;

Francesco Nesa, MD; Umberto Cortinovis, MD; Laura Sala, MD; Lorenza Di Guardo, MD;

Carolina Cimminiello, MD; Michele Del Vecchio, MD; Barbara Valeri, MD; and Mario Santinami, MD

J Natl Compr Canc Netw 2021;19(10):1165-1173

eTable 1: Results of Univariable Cox Models for OS and DFS 


\section{eTable 1. Results of Univariable Cox Models for OS and DFS}

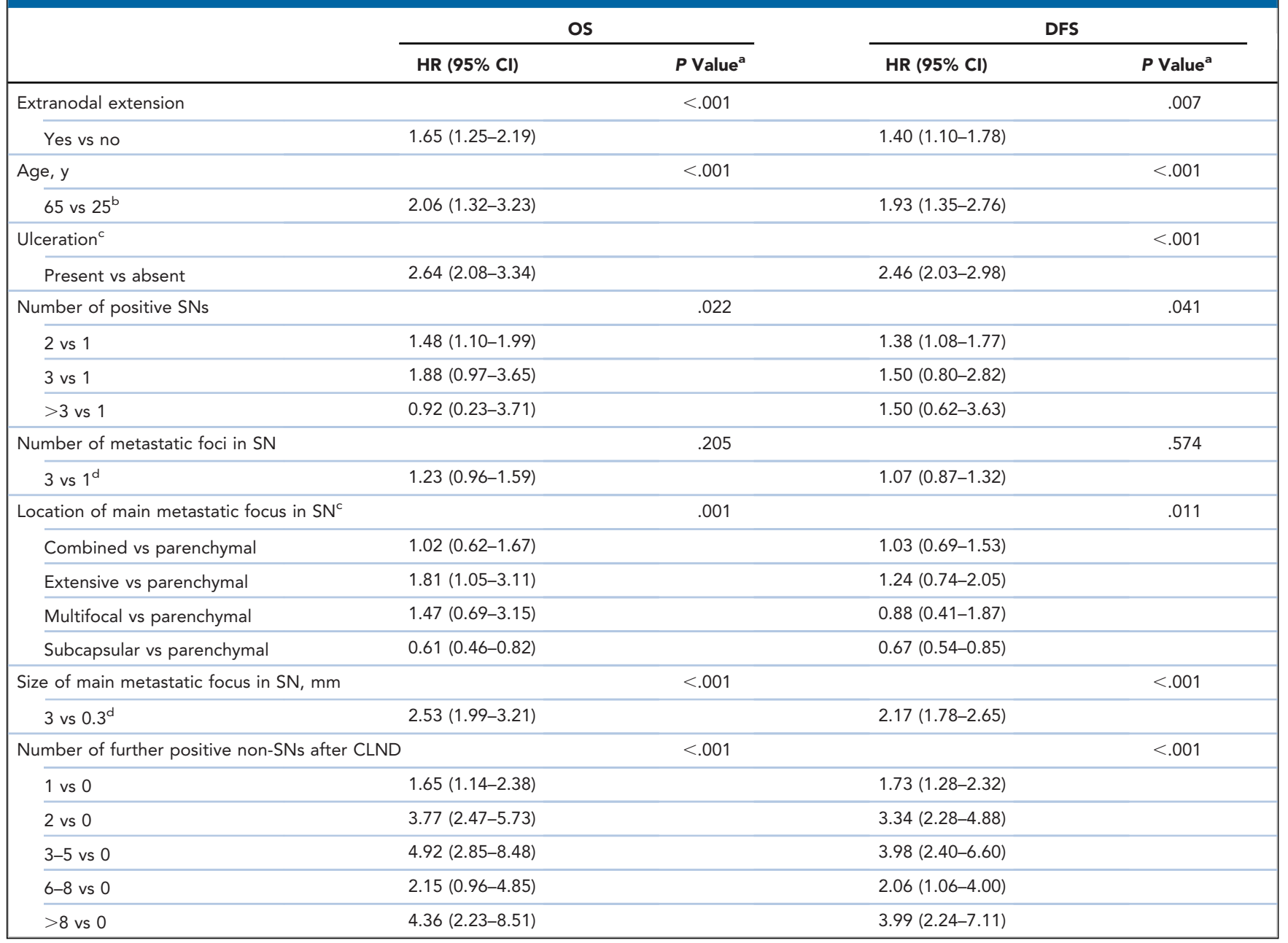

Abbreviations: CLND, completion lymph node dissection; DFS, disease-free survival; HR, hazard ratio; OS, overall survival; SN, sentinel node.

${ }^{a}$ Calculated using a 2-sided Wald test.

${ }^{b}$ Modeled as restricted cubic spline.

'Patients in whom ulceration and location of the main metastatic focus in the SN were not evaluable were excluded from the analyses.

${ }^{\mathrm{d}}$ Modeled as restricted cubic spline; values representing $3 \mathrm{rd}$ and 1 st quartile. 